\title{
Flag-no-square triangulations and Gromov boundaries in dimension 3
}

\author{
Piotr Przytycki ${ }^{1}$ and Jacek Świątkowski ${ }^{2}$
}

\begin{abstract}
We describe an infinite family of 3-dimensional topological spaces, which are homeomorphic to boundaries of certain word-hyperbolic groups. The groups are right-angled hyperbolic Coxeter groups, whose nerves are flag-no-square triangulations of 3-dimensional manifolds. We prove that any 3-dimensional polyhedral complex (in particular, any 3-manifold) can be triangulated in a flag-no-square way.
\end{abstract}

Mathematics Subject Classification (2000). 20F67; 57Q15, 20F65, 20 F55.

Keywords. Word-hyperbolic group, Gromov boundary, flag-no-square triangulation.

\section{Introduction}

The Gromov boundary of a word-hyperbolic group is known to be a compact finite dimensional metrizable space. It is connected unless the group essentially splits (as an amalgamated free product or as an HNN-extension) over a finite subgroup. When the boundary is connected and distinct from $S^{1}$, it has no local cut points unless the group essentially splits over a two-ended subgroup [1].

Not many explicit topological spaces are known to be homeomorphic to the boundary of a word-hyperbolic group. Restricting to the case of indecomposable groups, we may ask for such spaces that are connected and have no local cut points. In dimension 1 there are precisely two possibilities: the Sierpiński carpet $M_{1,2}$ and the Menger curve $M_{1,3}$ [16]. Surprisingly little is known in dimensions larger than 1 . The only known examples, apart from spheres $S^{n}$ and Sierpiński compacta $M_{n, n+1}$, are the Menger universal compacta $M_{2,5}$ and $M_{3,7}$ [8], and certain 2-dimensional compacta $\Pi_{p}$ called Pontriagin surfaces [7].

Spheres (and Sierpiński compacta) occur as boundaries of fundamental groups of hyperbolic manifolds (with totally geodesic boundary). The Menger compacta $M_{2,5}$ and $M_{3,7}$ occur as boundaries of right-angled hyperbolic buildings of dimension 3

\footnotetext{
${ }^{1}$ The first author was partially supported by MNiSW grant N201 003 32/0070, MNiSW grant N201 012 32/0718, and the Foundation for Polish Science.

${ }^{2}$ The second author was partially supported by MNiSW grants 2 P03A 01725 and N201 012 32/0718.
} 
and 4, respectively. Consequently, they are the boundaries of discrete cocompact automorphism groups of such buildings. Pontriagin surfaces $\Pi_{p}$ (where $p$ is a prime) are characterized by $\operatorname{dim}_{Q}\left(\Pi_{p}\right)=\operatorname{dim}_{Z_{q}}\left(\Pi_{p}\right)=1$ for any prime $q$ distinct from $p$, and $\operatorname{dim}_{Z_{p}}\left(\Pi_{p}\right)=2$, where $\operatorname{dim}_{G}$ is the cohomological dimension for coefficients $G$. They are the boundaries of certain right-angled hyperbolic Coxeter groups.

Dranishnikov's method [7] combined with a recent result of Fischer [9] yields also the Pontriagin sphere (which is different from the Pontriagin surfaces $\Pi_{p}$ ). It occurs as the boundary of all right-angled hyperbolic Coxeter groups whose nerves are closed orientable surfaces (see Remarks 3.6 and $4.4(1)$ ).

In this paper we describe another family of topological spaces, in dimension 3, that are the boundaries of right-angled hyperbolic Coxeter groups. These are some of the trees of manifolds (named so in [10]) introduced by Jakobsche in [12]. Apart from being connected, these spaces are homogeneous, and thus have no local cut points. Moreover, they are (examples of) Cantor manifolds, which means that no subset of codimension 2 or more (i.e., of dimension $\leq 1$ ) separates them.

Trees of manifolds are defined as inverse limits of appropriate systems of iterated connected sums of manifolds; see Section 3. They generalize the Pontriagin sphere which is obtained in this way out of 2-dimensional tori. By the already mentioned result of Fischer [9], certain trees of manifolds occur as boundaries of those rightangled hyperbolic Coxeter groups whose nerves are manifolds, PL-triangulated in a flag-no-square way. An essential part of this paper is the construction of flag-nosquare triangulations for any 3-dimensional polyhedral complex, in particular for any 3 -dimensional PL-manifold. This construction uses certain properties of the 600-cell, and occupies Section 2.

Our approach cannot be widely extended to higher dimensions. It is known for example that 4-dimensional homology spheres do not admit flag-no-square triangulations (see Section 2.2 in [14] and Theorem 5.6 in the appendix). As a consequence, no manifold in dimension above 4 has a flag-no-square PL-triangulation. The question of which 4-manifolds admit flag-no-square triangulations is (according to our knowledge) an open problem. We describe a class of such 4-manifolds in Remark 4.4 (2).

On the other hand, our construction of flag-no-square triangulations in dimension 3 waits for other applications. For example, it might be useful for constructing word-hyperbolic groups whose boundaries have exotic cohomological dimensions for various coefficients, in the spirit of [7].

We close the paper with an appendix containing the summary of what is known about flag-no-square triangulations.

We are grateful to Tadeusz Januszkiewicz for posing the problem of flag-no-square subdivision of 3-dimensional simplicial complexes and for motivating us. We thank Paweł Krupski for pointing out to us various topological references. 


\section{Flag-no-square subdivision in dimension 3}

In this section we show that any simplicial complex of dimension $\leq 3$ can be subdivided so as to satisfy the flag-no-square property. We recall the definition of the flag-no-square property, and briefly outline its geometric role, in the appendix to this paper.

Consider the 600-cell, the convex regular 4-polytope with Schläfli symbol $\{3,3,5\}$ (see, e.g., [3]). Denote by $X_{600}$ the boundary of the 600-cell, a 3-dimensional simplicial polyhedron homeomorphic to the 3-dimensional sphere. It consists of 600 3 -simplices and has 120 vertices. Its vertex links are icosahedra and edge links are pentagons. We will only exploit the combinatorial simplicial structure of $X_{600}$.

A starting point for our construction of flag-no-square subdivisions is the following.

Lemma 2.1. $X_{600}$ satisfies the flag-no-square property.

Proof. We present an argument that refers to Moussong's characterization of wordhyperbolicity for right-angled Coxeter groups (see Corollary 5.3 in the appendix). Consider the 120-cell $P_{120}$, which is a convex regular 4-dimensional polytope that is simple and whose 3-dimensional faces are dodecahedra (see, e.g., [3]). Realize $P_{120}$ as a right-angled convex polytope in the hyperbolic space $\mathbb{H}^{4}$ (see [2], the tile of the honeycomb $\{5,3,3,4\}$ ), and consider the group $W$ generated by reflections with respect to the 3-dimensional faces of $P . W$ is then a right-angled Coxeter group which acts properly discontinuously and cocompactly on $\mathbb{H}^{4}$ (compare Theorem 6.4.3 in [6]), and hence it is word-hyperbolic. Moreover, the nerve of $W$ is the simplicial complex dual to the boundary complex $X_{120}=\partial P_{120}$ (Example 7.14 in [6]), and thus it is isomorphic to $X_{600}$ (see [3]). By the above mentioned Moussong's characterization, $X_{600}$ is flag-no-square, and the lemma follows.

The lemma can be also proved in an elementary way, by the direct inspection of the combinatorial ball of radius 2 around a vertex in $X_{600}$. We omit the details.

Before constructing flag-no-square subdivisions in dimension 3 , we recall the analogous construction in dimension 2, due to Dranishnikov [7]. Let $Z_{10}$ be the subcomplex of the boundary triangulation of the icosahedron which is the span of the vertices at distance at most 1 (in the 1-skeleton) from a fixed 2-dimensional simplex. Clearly, $Z_{10}$ is topologically a 2-dimensional disc. We call true vertices of $Z_{10}$ those vertices which belong to exactly two different 2-dimensional simplices of $Z_{10}$. There are three such vertices and they lie on the boundary of $Z_{10}$.

Definition 2.2. The special subdivision of a 2 -simplex $\Delta$ is the subdivision isomorphic to $Z_{10}$, where vertices of $\Delta$ correspond to true vertices of $Z_{10}$. We denote it by $\Delta^{*}$. For any 2-dimensional simplicial complex $Y$, the special subdivision $Y^{*}$ is obtained by taking the first barycentric subdivision of the 1-skeleton of $Y$, followed by the special subdivision of every 2 -simplex of $Y$. 
We recall from [7] the following.

Lemma 2.3. Let $Y$ be a 2-dimensional simplicial complex. Then its special subdivision $Y^{*}$ satisfies the flag-no-square property.

Now we turn to looking closely at the combinatorial ball around a 3-simplex in $X_{600}$. Its properties together with the flag-no-square property of $X_{600}$ are crucial for the later construction in this section. Fix a 3-dimensional simplex $\Delta_{0}$ in $X_{600}$. Consider the subcomplex

$$
B_{1}\left(\Delta_{0}\right)=\operatorname{span}\left\{v \in X_{600}^{(0)}: \operatorname{dist}\left(v, \Delta_{0}\right) \leq 1\right\}
$$

Denote by $\partial B_{1}\left(\Delta_{0}\right)$ the subcomplex of $B_{1}\left(\Delta_{0}\right)$ spanned by the vertices not in $\Delta_{0}$.

Lemma 2.4. Every simplex in $\partial B_{1}\left(\Delta_{0}\right)$ is contained in a simplex intersecting $\Delta_{0}$.

Proof. If the simplex of $\partial B_{1}\left(\Delta_{0}\right)$ is a vertex, the assertion is trivial. If the simplex is an edge $x y$ and $x^{\prime}, y^{\prime}$ are neighbors in $\Delta_{0}$ of $x, y$ respectively, then the flag-nosquare property of $X_{600}$ implies, without loss of generality, that $x y^{\prime}$ is an edge, so $x y$ belongs to a triangle $x y y^{\prime}$ intersecting $\Delta_{0}$.

Now if $x y z$ is a triangle in $\partial B_{1}\left(\Delta_{0}\right)$ then let $x^{\prime}, y^{\prime}$ denote vertices in $\Delta_{0}$ forming triangles $z x x^{\prime}, z y y^{\prime}$ guaranteed by the previous step. If $x^{\prime}=y^{\prime}$ then the assertion follows by flagness of $X_{600}$. If not, consider the cycle $x y y^{\prime} x^{\prime} x$ and note that the flag-no-square condition yields, without loss of generality, that $x y^{\prime}$ is an edge, so $x y z y^{\prime}$ is a simplex.

Finally, we need to show that $\partial B_{1}\left(\Delta_{0}\right)$ contains no 3-simplex. If not, let $x, y$, $z, t$ be vertices spanning a 3-simplex of $\partial B_{1}\left(\Delta_{0}\right)$. Let $u, w$ be the vertices in $\Delta_{0}$ forming 3-simplices $x y z u$ and $y z t w$ guaranteed by the previous step. If $u=w$ then flagness of $X_{600}$ yields that $x y z t u w$ is a 4-simplex of $X_{600}$, a contradiction. If $u \neq w$, consider the cycle $u w t x u$ and note that the flag-no-square condition yields, without loss of generality, that $x w$ is an edge, so $x y z u w$ is a 4-simplex of $X_{600}$, which gives again a contradiction.

The above lemma allows one to describe both complexes $B_{1}\left(\Delta_{0}\right)$ and $\partial B_{1}\left(\Delta_{0}\right)$ more precisely.

Lemma 2.5. (1) $B_{1}\left(\Delta_{0}\right)$ is topologically a 3-ball, and $\partial B_{1}\left(\Delta_{0}\right)$ is its boundary sphere.

(2) $\partial B_{1}\left(\Delta_{0}\right)$ is simplicially isomorphic to the special subdivision $\left(\partial \sigma^{3}\right)^{*}$ of the boundary of a 3-simplex.

(3) Under the canonical identification of $\partial B_{1}\left(\Delta_{0}\right)$ with $\left(\partial \sigma^{3}\right)^{*}$, if vertices $w_{1}, w_{2} \in \partial B_{1}\left(\Delta_{0}\right)$ are contained in the same proper face $\tau$ of $\sigma^{3}$, then there is a vertex $v_{0} \in \Delta_{0}$ at distance 1 from both $w_{1}$ and $w_{2}$. 
Proof. By Lemma 2.4 and the fact that in $X_{600}$ each simplex is contained in a 3-simplex, $\partial B_{1}\left(\Delta_{0}\right)$ is the union of the faces disjoint with $\Delta_{0}$ of the 3 -simplices intersecting $\Delta_{0}$. Thus $B_{1}\left(\Delta_{0}\right)$ is the union of the 3-simplices that intersect $\Delta_{0}$. We describe it gradually, starting from the position of the 3-simplices with 2-dimensional intersection with $\Delta_{0}$ and ending with the ones with 0 -dimensional intersection.

Denote the vertices of $\Delta_{0}$ by $v_{i}, i=1,2,3,4$. First consider four 3-dimensional simplices $\Delta_{i}, i=1,2,3,4$, of $X_{600}$, which have 2-dimensional intersection with $\Delta_{0}$ $\left(v_{i} \notin \Delta_{0} \cap \Delta_{i}\right.$ ). Denote by $a_{i}, i=1,2,3,4$, the vertices in corresponding $\Delta_{i}$ not contained in $\Delta_{0}$, thus being vertices in $\partial B_{1}\left(\Delta_{0}\right)$. Observe that for $i \neq j$ we have $a_{i} \neq a_{j}$. Otherwise $a_{i}$ would be a neighbor of all vertices of $\Delta_{0}$, and by flagness we would have a 4-simplex, contradiction.

Now consider twelve 3-dimensional simplices $\Delta_{i j}, i, j=1,2,3,4, i \neq j$, such that $\Delta_{i j} \cap \Delta_{0}$ is 1-dimensional, $\Delta_{i j} \cap \Delta_{0}=\Delta_{i} \cap \Delta_{j}, \Delta_{i j} \cap \Delta_{i}$ is 2-dimensional. In each $\Delta_{i j}$ there is one edge disjoint with $\Delta_{0}$, whose one vertex is $a_{i}$ and second is a common vertex with $\Delta_{j i}$. Denote this common vertex by $a_{i j}(i<j)$. Observe that for $\{i, j\} \neq\{k, l\}$ (and any $m$ ) we have $a_{i j} \neq a_{k l}\left(a_{i j} \neq a_{m}\right)$. Otherwise, if $a_{i j}=a_{k l}$, then $a_{i j}$ is a neighbor of at least three different vertices of $\Delta_{0}$ and by flagness it is one of the $a_{m}$. If $a_{i j}=a_{m}$, then $m \notin\{i, j\}$, hence $a_{i j}$ is a neighbor of all vertices of $\Delta_{0}$, and by flagness we have a 4 -simplex, contradiction.

Altogether, the union $\Delta_{0} \cup \bigcup \Delta_{i} \cup \bigcup \Delta_{i j}$ is homeomorphic to the 3-ball, and its intersection with $\partial B_{1}\left(\Delta_{0}\right)$ is simplicially isomorphic to the first barycentric subdivision of the 1-skeleton of the 3-dimensional simplex.

Finally, consider the rest of the 3-dimensional simplices of $X_{600}$ having non-empty intersection with $\Delta_{0}$, grouped into the following four complexes. Let

$$
C_{i}=\bigcup\left\{\Delta: \Delta \text { is a 3-dimensional simplex of } B_{1}\left(\Delta_{0}\right), \Delta \cap \Delta_{0}=v_{i}\right\},
$$

for $i=1,2,3,4$. Recall that the star of the vertex $v_{i}$ is the cone over the icosahedron. Observe that $C_{i}$ consists of exactly half of the 3-dimensional simplices of this star. To see this, we check which simplices of this star do not belong to $C_{i}$. First, $C_{i}$ does not contain $\Delta_{0}$. It also does not contain any of $\Delta_{j}, j \neq i$, since these have 2-dimensional intersection with $\Delta_{0}$. Finally, $C_{i}$ does not contain the simplices $\Delta_{k l}$, $k, l \neq i$, since they have 1-dimensional intersection with $\Delta_{0}$. It particular, the triangles corresponding to these 3 -simplices in the link of $v_{i}$ are the triangles which have a nonempty intersection with the triangle corresponding to $\Delta_{0}$. Thus the link of $v_{i}$ in $C_{i}$ (which is isomorphic to the intersection of $C_{i}$ with $\partial B_{1}\left(\Delta_{0}\right)$ ) is simplicially isomorphic to $Z_{10}$. It is glued to the previously constructed part of $\partial B_{1}\left(\Delta_{0}\right)$ in such a way that true vertices of the new part are glued to the vertices $a_{j}, j \neq i$.

To complete the proof of part (2) we now only need the following observation. A vertex $c_{i} \in C_{i}^{(0)}$ different from $v_{i}$, from $a_{m}$ for $m \neq i$ and from $a_{k l}$ for $i \notin\{k, l\}$ does not coincide with any $a_{m}, a_{k l}$ or other $c_{j} \in C_{j}^{(0)} \backslash\left\{v_{j}\right\}$. Otherwise, if $c_{i}=c_{j}$, then (since $i \neq j$ ) $c_{i}$ is a neighbor of two different vertices of $\Delta_{0}$. Hence by flagness it is contained in a 3-simplex with at least 1-dimensional intersection with $\Delta_{0}$, and 
thus it is one of $a_{m}$ or $a_{k l}$. If $c_{i}=a_{k l}$ then (since $i \notin\{k, l\}$ ) it is actually a neighbor of three different vertices of $\Delta_{0}$, hence by flagness it one of the $a_{m}$. If $c_{i}=a_{m}$, then $m=i$, hence $c_{i}$ is a neighbor of all vertices of $\Delta_{0}$ and again flagness yields a contradiction. This completes the proof of part (2).

Part (1) follows by observing that each $C_{i}$ is topologically a 3-ball, glued to the previously constructed part of $B_{1}\left(\Delta_{0}\right)$ (which is itself a 3-ball) along a 2-disc contained in the boundary $\partial C_{i}$. The subcomplex $\partial B_{1}\left(\Delta_{0}\right)$ is the boundary sphere of the so obtained 3-ball.

Part (3) easily follows from the above description of $\partial B_{1}\left(\Delta_{0}\right)$.

In order to extend special subdivisions to dimension 3, we use the complement of the ball $B_{1}\left(\Delta_{0}\right)$ in $X_{600}$. More precisely, denote by $X_{543}$ the 3-dimensional subcomplex of $X_{600}$ which is the closure of $X \backslash B_{1}\left(\Delta_{0}\right)$. In other words, $X_{543}$ is the union of all 3-simplices of $X_{600}$ not contained in $B_{1}\left(\Delta_{0}\right)$. (The number 543 in the subscript is the number of 3 -simplices in this subcomplex.)

Remark 2.6. It follows from Lemma 2.5 that $X_{543}$ is a 3-ball and its boundary sphere is simplicially isomorphic to $\left(\partial \sigma^{3}\right)^{*}$.

Lemma 2.7. (1) $X_{543}$ is a full subcomplex of $X_{600}$.

(2) $X_{543}$ satisfies the flag-no-square property.

Proof. To prove (1), consider a collection of vertices in $X_{543}$ spanning a simplex $\tau$ of $X_{600}$. If at least one of those vertices is interior in $X_{543}$ then clearly $\tau$ is a simplex of $X_{543}$. Otherwise, suppose that all simplices in the collection belong to the boundary sphere $\partial B_{1}\left(\Delta_{0}\right)$. Since, by definition, the subcomplex $\partial B_{1}\left(\Delta_{0}\right)$ is full in $X_{600}$, assertion (1) follows.

Since the flag-no-square property is inherited by full subcomplexes (Lemma 5.1 in the appendix), assertion (2) follows from Lemma 2.1.

Now we are ready to define special subdivision for 3-dimensional complexes.

Definition 2.8. Given a simplicial complex $W$ with $\operatorname{dim} W \leq 3$, its special subdivision $W^{*}$ is the simplicial complex obtained by taking the special subdivision of the 2-skeleton $W^{(2)}$ followed by subdividing each 3-simplex $\sigma^{3}$ of $W$ so that it becomes isomorphic to $X_{543}$ and its subdivided boundary $\left(\partial \sigma^{3}\right)^{*}$ canonically identifies with $\partial B_{1}\left(\Delta_{0}\right)$.

To prove that special subdivision yields simplicial complexes that satisfy the flagno-square property, we need some preparatory results.

Lemma 2.9. Let $\Delta$ be a simplex of dimension 1, 2 or 3, and let $x y$ be an edge of the special subdivision $\Delta^{*}$. If the vertices $x, y$ are not both contained in a common proper face of $\Delta$, then at least one of them lies in the interior of $\Delta$. 
Proof. For dimension 1 and 2 the proof goes by inspection. For dimension 3, suppose that both $x$ and $y$ are contained in the boundary of $\Delta$. Identify $\Delta^{*}$ with $X_{543} \subset X_{600}$, and recall that its boundary $\partial B_{1}\left(\Delta_{0}\right)$ is by definition full in $X_{600}$. Thus the edge $x y$ is contained in the boundary too. Let $\tau$ be a proper face of $\Delta$ containing the edge $x y$. Then both $x$ and $y$ are contained in $\tau$, contradiction.

Lemma 2.10. Let $W$ be a simplicial complex with $\operatorname{dim} W \leq 3$ and let $U$ be a subcomplex of $W$. Then $U^{*}$ is a full subcomplex of $W^{*}$.

Proof. Let the vertices $x, y \in U^{*}$ form an edge $x y$ of $W^{*}$. Denote by $\Delta \in W$ the simplex of the lowest possible dimension such that $x y \subset \Delta^{*}$. By Lemma 2.9, at least one of the vertices, say $y$, belongs to the interior of $\Delta$. This implies that $\Delta \subset U$ and $x y \subset \Delta^{*} \subset U^{*}$.

For a triangle or a 3-simplex $\sigma$ of $W^{*}$ with vertices in $U^{*}$ the argument is the same. If $\Delta$ is a minimal simplex of $W$ such that $\sigma \subset \Delta^{*}$, then, by a version of Lemma 2.9 for 2-simplices and 3-simplices, at least one of the vertices $y$ of $\sigma$ belongs to the interior of $\Delta$, so $\Delta \subset U$ and $\sigma \subset \Delta^{*} \subset U^{*}$.

Lemma 2.11. Let $\Delta$ be a 3-dimensional simplex. Let $w, w_{1}, w_{2}$ be vertices of $\Delta^{*}$ and suppose $w$ is interior in $\Delta$. Suppose also that $w_{1}, w_{2}$ lie on the same 2-dimensional face of $\Delta$, and that there are edges in $\Delta^{*}$ between $w$ and $w_{1}$ and between $w$ and $w_{2}$. Then there is an edge between $w_{1}$ and $w_{2}$ in $\Delta^{*}$.

Proof. View $\Delta^{*}$ as $X_{543}$, the subcomplex of $X_{600}$. By Lemma 2.5 (3), there is a vertex $v_{0}$ in $\Delta_{0}$ whose distance from both $w_{1}$ and $w_{2}$ is equal to one. If $w_{1}$ and $w_{2}$ were not connected by an edge, then the opposite vertices of the cycle $w_{1} v_{0} w_{2} w w_{1}$ would not be connected by edges, contradicting the flag-no-square property of $X_{600}$.

Lemma 2.12. Let $\Delta$ be a 3-simplex and $\tau$ a simplex of dimension $\leq 3$ such that $\Delta \cap \tau$ is a nonempty proper face in both $\Delta$ and $\tau$. Then the special subdivision $Y=(\Delta \cup \tau)^{*}$ satisfies the flag-no-square property.

Proof. To prove flagness, consider any collection of vertices in $Y$ pairwise connected by edges. We claim that all these vertices belong to a single subdivided simplex $\Delta^{*}$ or $\tau^{*}$. Indeed, if one of the vertices, say $v$, is not in the intersection $\Delta \cap \tau$ then all vertices in the collection belong to the same subdivided simplex as $v$. Now, since each of the subdivided simplices $\Delta^{*}$ and $\tau^{*}$ is full in $Y$ (Lemma 2.10) and flag (Lemma 2.7 (2) or Lemma 2.3), the vertices from the collection span a simplex of $Y$.

To prove the flag-no-square property, suppose that we have a cycle of length four in $Y$. If the cycle is contained in $\Delta^{*}$ or in $\tau^{*}$, then we use the flag-no-square property of $X_{543}$ (Lemma $2.7(2)$ ), or Lemma 2.3. Otherwise, suppose that a vertex $x$ of the cycle does not belong to $\Delta^{*}$, and that a vertex $y$ does not belong to $\tau^{*}$. This means 
that $x$ and $y$ are opposite in the cycle and that the other two vertices, $u$ and $w$, of the cycle belong to $\Delta \cap \tau$. By Lemma 2.11, $u$ and $w$ are connected by an edge, which finishes the proof.

We are now ready to prove the main result of this section:

Proposition 2.13. Let $W$ be a 3-dimensional simplicial complex. Then its special subdivision $W^{*}$ is flag-no-square.

Proof. To prove flagness, consider a collection of vertices of $W^{*}$ pairwise connected by edges. If all these vertices are in $\left(W^{(2)}\right)^{*}$, the special subdivision of the 2-skeleton of $W$, then they are pairwise connected by edges in $\left(W^{(2)}\right)^{*}$ by the fact that $\left(W^{(2)}\right)^{*}$ is full in $W^{*}$ (Lemma 2.10). They span a simplex because $\left(W^{(2)}\right)^{*}$ is flag (Lemma 2.3). Otherwise, there is a vertex in the collection contained in the interior of a 3-simplex $\Delta$ of $W$. Consequently, all vertices in the collection are the vertices of $\Delta^{*}$. Now, since $\Delta^{*}$ is full in $W^{*}$ (Lemma 2.10), the vertices in the collection are pairwise connected by edges in $\Delta^{*}$. Since the latter is flag (Lemma $2.7(2)$ ), the vertices span a simplex of $\Delta^{*}$, which is also a simplex of $W^{*}$.

To prove the flag-no-square property, consider a cycle of length 4 in $W^{*}$. If all vertices of the cycle are in $\left(W^{(2)}\right)^{*}$ then, by Lemma 2.10 , the whole cycle is contained in $\left(W^{(2)}\right)^{*}$. Since the latter is flag-no-square (Lemma 2.3), there is an edge between some opposite vertices in the cycle.

If one vertex, say $v_{1}$, in the cycle $v_{1} v_{2} v_{3} v_{4} v_{1}$ in $W^{*}$ is interior in a 3 -simplex $\Delta$ of $W$, then $v_{2}$ and $v_{4}$ are together with $v_{1}$ in $\Delta^{*}$. If $v_{3}$ is also in $\Delta^{*}$, the whole cycle is in $\Delta^{*}$ (by fullness of $\Delta^{*}$ in $W^{*}$, see Lemma 2.10). Since $\Delta^{*}$ is flag-no-square, the cycle is as required. If $v_{3}$ is not contained in $\Delta$, it belongs to some simplex $\tau$ which is not a face of but shares a face with $\Delta$. Moreover, the whole cycle is contained in the union $\Delta \cup \tau$ (Lemma 2.10 again). Since the special subdivision of the latter is flag-no-square (Lemma 2.12), this finishes the proof.

Corollary 2.14. Every 3-dimensional polyhedron can be triangulated in a flag-nosquare fashion.

Proof. Consider any triangulation of the polyhedron and take its special subdivision.

\section{Trees of manifolds}

In this section we recall from [12] Jakobsche's definition of a family of spaces that we call (after Fischer and Guilbault [10]) trees of manifolds. We include an extension to the case of non-orientable manifolds due to P. Stallings [21]. We recall and/or derive some useful topological properties of these spaces. We also recall a theorem of Fischer [9] who proved that some trees of manifolds are CAT( 0 ) boundaries of the 
Davis-Vinberg complexes of certain right-angled Coxeter groups. We focus only on trees of manifolds, which appear in Fischer's theorem.

Theorem 3.1 (Jakobsche [12], [9], Stallings [21]). Let $L_{0} \stackrel{\alpha_{1}}{\longleftarrow} L_{1} \stackrel{\alpha_{2}}{\longleftarrow} L_{2} \stackrel{\alpha_{3}}{\longleftarrow} \ldots$ be an inverse sequence of connected closed $n$-manifolds $(n \geq 2)$ and $\mathscr{D}_{k}$ finite collections of disjoint collared discs in $L_{k}$ such that

(a) each $L_{k}$ is a connected sum of finitely many copies of $L_{0}$;

(b) each $\alpha_{k+1}$ is a homeomorphism over the set $L_{k} \backslash \bigcup\left\{\right.$ int $\left.D \mid D \in \mathscr{D}_{k}\right\}$;

(c) each $\alpha_{k+1}^{-1}(D)\left(D \in \mathscr{D}_{k}\right)$ is homeomorphic to a copy of $L_{0}$ with the interior of a collared disc removed;

(d) $\left\{\alpha_{j+1} \circ \alpha_{j+2} \circ \cdots \circ \alpha_{i}(D) \mid D \in \mathscr{D}_{i}, i \geq j\right\}$ is null and dense in $L_{j}$ for all $j$;

(e) $\alpha_{j+1} \circ \alpha_{j+2} \circ \cdots \circ \alpha_{i}(D) \cap$ bdy $D^{\prime}=\emptyset$ for all $D \in \mathscr{D}_{i}, D^{\prime} \in \mathscr{D}_{j}, i>j$.

Then the inverse limit

$$
\lim _{\longleftarrow}\left(L_{0} \stackrel{\alpha_{1}}{\longleftarrow} L_{1} \stackrel{\alpha_{2}}{\longleftarrow} L_{2} \stackrel{\alpha_{3}}{\longleftarrow} \cdots\right)
$$

depends only on $L_{0}$. This space is denoted by $X\left(L_{0},\left\{L_{0}\right\}\right)$.

The spaces $X\left(L_{0},\left\{L_{0}\right\}\right)$ are clearly connected and locally connected compact metric spaces. Jakobsche and Stallings show in [13], [12] and [21] the following less immediate properties of these spaces. Recall that, given a positive integer $m$, a topological space $X$ is $m$-homogeneous if for any two $m$-element subsets of $X$ there is a homeomorphism of this space which maps one set to the other.

Theorem 3.2 (Jakobsche, Stallings). (1) The topological dimension $\operatorname{dim} X\left(L_{0},\left\{L_{0}\right\}\right)$ is equal to $\operatorname{dim} L_{0}$.

(2) For every positive integer $m$ the space $X\left(L_{0},\left\{L_{0}\right\}\right)$ is $m$-homogeneous.

(3) If $L_{0}$ is a homology $n$-sphere, then $X\left(L_{0},\left\{L_{0}\right\}\right)$ is a cohomology n-manifold.

The above properties of trees of manifolds have the following further consequences, especially interesting in the context of boundaries of word-hyperbolic groups. Recall that a topological space $X$ is a Cantor manifold if no subset of $X$ of dimension $\leq \operatorname{dim} X-2$ separates $X$.

Corollary 3.3. If $\operatorname{dim} L_{0} \geq 2$, then the spaces $X\left(L_{0},\left\{L_{0}\right\}\right)$

(1) have no local cut points, and

(2) are Cantor manifolds.

Proof. Let $X=X\left(L_{0},\left\{L_{0}\right\}\right)$. By homogeneity, either $X$ has no local cut point or every point of $X$ is a local cut point. Suppose the latter holds. Since in any continuum the set of local cut points which are not of order 2 is countable ([22], (9.2), p. 61), it 
follows that each point of $X$ is of order 2, and hence it is homeomorphic to $S^{1}$ ([18], Theorem 6, p. 294). This contradiction proves (1).

Since any homogeneous continuum is a Cantor manifold [17], (2) follows from Theorem $3.2(2)$.

It turns out that sometimes the spaces $X\left(L_{0},\left\{L_{0}\right\}\right)$ are homeomorphic for different manifolds $L_{0}$. On the other hand, Jakobsche [12] shows how to distinguish certain trees of manifolds, up to homeomorphism, in dimension 3 . We recall briefly some details concerning these two issues. We denote by $M \# N$ the connected sum of manifolds $M$ and $N$.

Lemma 3.4. If $M^{\prime}=M \# N$ and $M=L \# N$, then the spaces $X\left(M^{\prime},\left\{M^{\prime}\right\}\right)$ and $X(M,\{M\})$ are homeomorphic.

Proof. Both spaces are easily seen to be homeomorphic to Jakobsche's space $X(L,\{L, N\})$ (see [12] for the definition).

In dimension 2, in view of the classification of surfaces, Lemma 3.4 implies the following.

Corollary 3.5. Let $F_{1}, F_{2}$ be closed surfaces different from the 2-sphere. If either both $F_{1}, F_{2}$ are orientable or both are non-orientable, then the spaces $X\left(F_{1},\left\{F_{1}\right\}\right)$ and $X\left(F_{2},\left\{F_{2}\right\}\right)$ are homeomorphic.

Remark 3.6. Note that if $F$ is an orientable closed surface, then the space $X(F,\{F\})$ is the well-known Pontriagin sphere. If $F$ is a non-orientable closed surface then $X(F,\{F\})$ is the Pontriagin surface $\Pi_{2}$ mentioned in the introduction (see [23]). The Pontriagin sphere is not homeomorphic to any of the Pontriagin surfaces $\Pi_{p}$, as its cohomological dimension for coefficients $Q$ equals 2 .

In the case of 3-manifolds, Lemma 3.4 and the argument as in the proof of Theorem 11.1 in [12] imply the following.

Proposition 3.7. (1) If $L_{0}, L_{0}^{\prime}$ are closed 3-manifolds different from the sphere, and the summands appearing in their prime decompositions coincide (the numbers of their occurrences do not have to match) then the spaces $X\left(L_{0},\left\{L_{0}\right\}\right)$ and $X\left(L_{0}^{\prime},\left\{L_{0}^{\prime}\right\}\right)$ are homeomorphic.

(2) Let $L_{0}, L_{0}^{\prime}$ be closed orientable 3-manifolds. Suppose that, in their prime decompositions, a certain summand of $L_{0}$ has different fundamental group from all summands of $L_{0}^{\prime}$. Then the spaces $X\left(L_{0},\left\{L_{0}\right\}\right)$ and $X\left(L_{0}^{\prime},\left\{L_{0}^{\prime}\right\}\right)$ are not homeomorphic.

Now let us state the announced theorem of Fischer. For the definition of the Davis-Vinberg complex of a Coxeter group see [4]. By bdy $(\Gamma)$ we denote the CAT $(0)$ boundary of the Davis-Vinberg complex of the group $\Gamma$. 
Theorem 3.8 (Fischer [9]). If the nerve $N$ of a right-angled Coxeter group $\Gamma$ is a connected closed (orientable or not) PL-manifold, then bdy $(\Gamma)$ is homeomorphic to Jakobsche's $X(|N|,\{|N|\})$ space.

\section{The main theorem}

In this section we formulate and prove the main result of the paper, Theorem 4.1. We comment on the algebraic consequences for groups appearing in Theorem 4.1, implied by topological properties of 3-dimensional trees of manifolds. Finally, we show the consequences of arguments similar to ours for dimensions different than 3 .

Recall that all 3-manifolds are triangulable, all their triangulations are PL, and any two triangulations of a fixed 3-manifold are PL-equivalent [19].

Theorem 4.1. Let $N$ be a connected, closed 3-dimensional manifold. Then there exists a right-angled Coxeter group $\Gamma$ which is word-hyperbolic and its Gromov boundary is homeomorphic to Jakobsche's $X(N,\{N\})$ space.

Proof. Take any PL-triangulation of $N$. Let $N^{*}$ be its special subdivision, as defined in Section 2. Since $N^{*}$ is flag-no-square (Proposition 2.13), the right-angled Coxeter group $\Gamma$ whose nerve is $N^{*}$ is word-hyperbolic (Lemma 5.3 in the appendix). The Gromov boundary of $\Gamma$ is homeomorphic to the CAT $(0)$ boundary of the DavisVinberg complex of $\Gamma$. Thus, due to Fischer's Theorem 3.8, the Gromov boundary of $\Gamma$ is homeomorphic to Jakobsche's $X(N,\{N\})$ space.

Remark 4.2. Since the space $X(N,\{N\})$ is connected and has no local cut point (Lemma 3.3 (1)), it follows from a result of Bowditch [1] that $\Gamma$ as in Theorem 4.1 is JSJ-indecomposable (i.e., it does not split as an amalgamated free product or an HNN extension over a finite or 2-ended subgroup). Moreover, since $X(N,\{N\})$ is a Cantor manifold of topological dimension 3 (Corollary 3.3 (2) and Theorem 3.2 (1)), it is not separated by a Cantor set or a circle. Consequently, the corresponding group $\Gamma$ does not split over an undistorted virtually free or virtually surface subgroup.

The argument as in the proof of Theorem 4.1 clearly gives the following.

Corollary 4.3. If $N$ is a connected closed manifold (of arbitrary dimension greater than 1) which admits a flag-no-square PL-triangulation, then the space $X(N,\{N\})$ is homeomorphic to the Gromov boundary of a word-hyperbolic right-angled Coxeter group.

Remark 4.4. (1) The above corollary, together with Dranishnikov's Lemma 2.3, shows that there are word-hyperbolic groups whose boundaries are homeomorphic to the Pontriagin sphere. 
(2) In dimension 4 the only examples known to us of manifolds $N$ as in Corollary 4.3 are the following. Consider the regular simplicial tesselation of the hyperbolic space $\mathbb{H}^{4}$ with all vertex links isomorphic to $X_{600}$ - the boundary complex of the 600-cell. It is not hard to show (using Lemma 2.1) that this tesselation is flag-nosquare. The automorphism group $G$ of this tesselation is a Coxeter group, hence it is residually finite. In particular, $G$ contains torsion-free subgroups $G^{\prime}$ for which the quotients have arbitrarily large injectivity radius. If the injectivity radius is large enough, the quotient (viewed as a simplicial manifold) is flag-no-square. It is PL regardless of the injectivity radius.

(3) It follows from a result of Januszkiewicz and Światkowski [14] (see also Corollary 5.7 (2) in the appendix) that no closed manifold of dimension $\geq 5$ admits a flag-no-square triangulation. Thus Corollary 4.3 gives no trees of manifolds in dimensions above 4 as Gromov boundaries of word-hyperbolic groups.

\section{Appendix: Flag-no-square triangulations}

The aim of this appendix is to survey the subject of flag-no-square triangulations, and to formulate some open questions. All we know about this we have learnt from or discovered together with Tadeusz Januszkiewicz.

For completeness, we start with definitions.

A simplicial complex $X$ is $f l a g$ if any finite subset of its vertices pairwise connected by edges spans a simplex of $X$.

A cycle in $X$ is a subcomplex homeomorphic to the circle $S^{1}$. The length of a cycle is the number of edges in this cycle. A diagonal of a cycle is an edge connecting any two non-consecutive vertices in this cycle.

A simplicial complex $X$ is said to satisfy the flag-no-square condition if $X$ is flag and any cycle of length 4 in $X$ has a diagonal (equivalently, there is no full cycle of length 4 in $X$ ). For brevity we say that $X$ is flag-no-square. The same condition is also known as "no empty square" condition.

The following two properties of the flag-no-square condition are immediate but useful consequences of the definition. The second property follows from the first by the fact that links in a flag simplicial complex are full subcomplexes of it.

Lemma 5.1. Let $X$ be a flag-no-square simplicial complex. Then

(1) any full subcomplex of $X$ is flag-no-square;

(2) links of $X$ are flag-no-square.

The flag-no-square condition was introduced and studied in the context of cubical structures on 3-manifolds by L. Siebenmann. Its importance for the geometry of cubical complexes comes from the following observation by Gromov ([11], p. 123). 
Proposition 5.2. Let $X$ be a cubical complex with flag-no-square links. Then $X$ admits a negatively curved piecewise hyperbolic metric. In particular, the fundamental group of $X$ is word-hyperbolic.

This proposition implies in particular the following (see [20] or [5]).

Corollary 5.3. A right-angled Coxeter group is word-hyperbolic iff its nerve is a flag-no-square simplicial complex.

In [15] the flag-no-square condition has been reintroduced under the name 5-largeness and put in the wider context of combinatorial "metric" conditions for simplicial complexes. In this approach, we say that a simplicial complex $X$ is $k$-large (for an integer $k \geq 4$ ) if $X$ is flag and any cycle $\gamma$ in $X$, with length $|\gamma|$ satisfying $3<|\gamma|<k$, has a diagonal (equivalently, any full cycle in $X$ has length $\geq k$ ).

It turns out that 6-largeness of links (called briefly local 6-largeness) is a condition that resembles nonpositive curvature, sharing many consequences with the latter. For example, any compact locally 6-large simplicial complex is aspherical and its fundamental group is semi-hyperbolic. Furthermore, the fundamental group of any compact locally 7-large simplicial complex is word-hyperbolic.

Local 5-largeness is a weaker condition and does not lead to phenomena related to nonpositive curvature. For example, boundaries of the icosahedron and the 600cell are 5-large (and thus also locally 5-large) triangulations of the spheres $S^{2}$ and $S^{3}$. However, this condition may be still viewed as a kind of upper curvature bound. There are reasons to expect topological consequences of local 5-largeness similar to those of nonpositive curvature, in higher dimensions.

In the remaining part of this appendix we will use the term "5-large" instead of "flag-no-square".

In low dimensions there are no topological restrictions for 5-largeness. Namely, the following fact was observed by Dranishnikov [7].

Proposition 5.4. Any 2-dimensional polyhedron admits a 5-large triangulation.

In this paper we have strengthened this result by showing:

Proposition 5.5 (Corollary 2.14). Any polyhedron of dimension $\leq 3$ admits a 5-large triangulation.

Starting from dimension 4, there are topological obstructions for 5-largeness. They concern, among others, the so-called generalized homology spheres (GHS).

A simplicial complex $N$ is a generalized homology sphere of dimension $k$ if it has the same homology as the sphere $S^{k}$ and if the link of each simplex in $N$ is a generalized homology sphere of appropriate dimension. Along the lines of Section 2.2 in [14] the following result is proved. 
Theorem 5.6. A generalized homology sphere of dimension $k \geq 4$ is never 5-large.

Recall that links of PL-triangulations of manifolds are spheres. Links of other triangulations of manifolds may be not spheres, but they are generalized homology spheres. In particular, all triangulations of spheres and homology spheres are GHS. Moreover, links in any triangulation of a manifold are GHS. In view of these facts Theorem 5.6 has the following consequences.

Corollary 5.7. (1) No triangulation of a 4-dimensional homology sphere is 5-large. In particular, no triangulation of the sphere $S^{4}$ is 5-large.

(2) No triangulation of a manifold of dimension $n \geq 5$ is 5-large.

In contrast with the above result, constructions in [14] and [15] give many examples of compact 5-large pseudomanifolds, in arbitrary dimensions. In fact, the examples constructed there are 6-large, and thus aspherical. Taken as nerves, those examples yield word-hyperbolic right-angled Coxeter groups with arbitrarily large virtual cohomological dimension [14].

Coming back to manifolds, the only examples known to us of closed simplicial flag-no-square 4-manifolds are those described in Remark 4.4(2) in this paper.

We finish by posing some open problems that we find intriguing in the perspective of viewing local 5-largeness as a certain kind of upper curvature bound.

Questions 5.8. (1) Is every 5-large (or locally 5-large) 4-dimensional simplicial manifold aspherical?

(2) Is every 5-large (or locally 5-large) $n$-dimensional simplicial pseudomanifold, for $n \geq 4$, aspherical?

(3) Find any restrictions for polyhedra in dimensions $\geq 4$ to admit a 5-large or locally 5-large triangulation.

\section{References}

[1] B. H. Bowditch, Cut points and canonical splittings of hyperbolic groups. Acta Math. 180 (1998), 145-186. Zbl 0911.57001 MR 1638764

[2] H. S. M. Coxeter, Regular honeycombs in hyperbolic space. In Proceedings Internat. Congr. Mathematicians (Amsterdam, 1954), vol. III, Noordhoff N.V., Groningen; NorthHolland , Amsterdam 1956, 155-169. Zbl 0073.36603 MR 0087114

[3] H. S. M. Coxeter, Regular polytopes. Methuen and Co., London 1948. Zbl 0031.06502 MR 0027148

[4] M. W. Davis, Groups generated by reflections and aspherical manifolds not covered by Euclidean space. Ann. of Math. (2) 117 (1983), 293-324. Zbl 0531.57041 MR 690848

[5] M. W. Davis, Nonpositive curvature and reflection groups. In Handbook of geometric topology, Elsevier, Amsterdam 2002, 373-422. Zbl 0998.57002 MR 1886674 
[6] M. W. Davis, The geometry and topology of Coxeter groups. London Math. Soc. Monographs 32, Princeton University Press, Princeton, NJ, 2008. Zbl 1142.20020 MR 2360474

[7] A. N. Dranishnikov, Boundaries of Coxeter groups and simplicial complexes with given links. J. Pure Appl. Algebra 137 (1999), 139-151. Zbl 0946.20020 MR 1684267

[8] J. Dymara and D. Osajda, Boundaries of right-angled hyperbolic buildings. Fund. Math. 197 (2007), 123-165. Zbl 05221117 MR 2365885

[9] H. Fischer, Boundaries of right-angled Coxeter groups with manifold nerves. Topology 42 (2003), 423-446. Zbl 1025.57040 MR 1941443

[10] H. Fischer and C. R. Guilbault, On the fundamental groups of trees of manifolds. Pacific J. Math. 221 (2005), 49-79. Zbl 1097.55011 MR 2194145

[11] M. Gromov, Hyperbolic groups. In Essays in group theory, Math. Sci. Res. Inst. Publ. 8, Springer-Verlag, New York 1987, 75-263. Zbl 0634.20015 MR 0919829

[12] W. Jakobsche, Homogeneous cohomology manifolds which are inverse limits. Fund. Math. 137 (1991), 81-95. Zbl 0727.57018 MR 1113561

[13] W. Jakobsche, The Bing-Borsuk conjecture is stronger than the Poincare conjecture. Fund. Math. 106 (1980), 127-134. Zbl 0362.54029 MR 580590

[14] T. Januszkiewicz and J. Świątkowski, Hyperbolic Coxeter groups of large dimension. Comment. Math. Helv. 78 (2003), 555-583. Zbl 1068.20043 MR 1998394

[15] T. Januszkiewicz and J. Świątkowski, Simplicial nonpositive curvature. Publ. Math. Inst. Hautes Études Sci. 104 (2006), 1-85. Zbl 1143.53039 MR 2264834

[16] M. Kapovich and B. Kleiner, Hyperbolic groups with low-dimensional boundary. Ann. Sci. École Norm. Sup. (4) 33 (2000), 647-669. Zbl 0989.20031 MR 1834498

[17] P. Krupski, Homogeneity and Cantor manifolds. Proc. Amer. Math. Soc. 109 (1990), 1135-1142. Zbl 0714.54035 MR 1009992

[18] K. Kuratowski, Topology. Vol. II, Academic Press, New York-London; PWN Polish Scientific Publishers, Warsaw 1968. MR 0259835

[19] E. E. Moise, Affine structures in 3-manifolds: V. The triangulation theorem and Hauptvermutung. Ann. of Math. (2) 56 (1952), 96-114. Zbl 0048.17102 MR 0048805

[20] G. Moussong, Hyperbolic Coxeter groups. Ph.D. thesis, The Ohio State University, Columbus 1988. http://www.ohiolink.edu/etd/view.cgi?osu1114437114

[21] P. R. Stallings, An extension of Jakobsche's construction of $n$-homogeneous continua to the nonorientable case. In Continua - with the Houston problem book, Lect. Notes in Pure and Appl. Math. 170, Marcel Dekker, New York 1995, 347-361. Zbl 0829.54024 MR 1326856

[22] G. T. Whyburn, Analytic topology. Amer. Math. Soc. Colloq. Publ. 28, Amer. Math. Soc., New York 1942. Zbl 0061.39301 MR 0007095

[23] R. F. Williams, A useful functor and three famous examples in topology. Trans. Amer. Math. Soc. 106 (1963), 319-329. Zbl 0113.37803 MR 0146832

Received July 20, 2007; revised September 2, 2008 
P. Przytycki, Institute of Mathematics of the Polish Academy of Sciences, Śniadeckich 8, 00-956 Warsaw, Poland

E-mail: pprzytyc@ mimuw.edu.pl

J. Świątkowski, Instytut Matematyczny, Uniwersytet Wrocławski, pl. Grunwaldzki 2/4, 50-384 Wrocław, Poland

E-mail: Jacek.Swiatkowski@math.uni.wroc.pl 